\title{
Exploration of Cognitive Functioning in a Pilot Sample of Childhood Cancer Patients in Egypt
}

\author{
Manal A. Shehata ${ }^{1}$, Mohamed Fawzy ${ }^{2}$, Mai S. Saleh ${ }^{3, *}$, Zeinab M. Monir ${ }^{1}$, Hanafy Ahmed ${ }^{2}$ \\ ${ }^{1}$ Child Health Department, National Research Center, 33 Elbehouth street Dokki, Giza, Egypt \\ ${ }^{2}$ Pediatric Oncology Department, National Cancer Institute, Cairo University, Fom El Khalig Square Kornish El Nil, Cairo, Egypt \\ ${ }^{3}$ Environmental and Occupational Medicine Department, National Research Center, 33 Elbehouth street, Dokki, Giza, Egypt \\ *Corresponding author: nouranomer@gmail.com
}

Received April 15, 2015; Revised April 28, 2015; Accepted May 07, 2015

\begin{abstract}
A subset of cancer survivors experience cognitive deficits that can last for many years after the completion of chemotherapy. The etiology of this problem is largely unknown, so the present study aimed to assess cognitive functioning in childhood patients with cancer and to investigate the proposed disposing factors including variables related to disease, treatment, and some socio-demographic characteristics. In a case control study parents of 67 cancer patients aged 8-12 years, completed the parent proxy report of PedsQL ${ }^{\mathrm{TM}} 3.0$ Cognitive Functioning Scale (Arabic versions), as well as a separate sheet for socio-demographic data. Control group consisted of 37 healthy subjects from the same age group were subjected to the same methodology for comparison. All patients under the study have successfully accomplished their treatment protocol and were in complete remission during the evaluation. Hematological malignancies represented $70.1 \%$ of the patients sample, with the highest proportion for ALL (52.2\%). Brain tumors represented $40 \%$ of the solid malignancies (29.9\% of the study patients). Cognitive functioning score was significantly lower in the solid group (69.6 \pm 37.3$)$ compared to the hematologic group $(85.1 \pm 22.2)(t=2.1, p=0.038)$. Cognitive functioning score was also lower in solid group versus control subjects ( $p$ $=0.047$ ), while it showed no significant difference between hematological malignancies and control group. Older age at diagnosis, urban residence, illiterate mothers, higher duration of treatment as well as long duration of hospital admission were associated with a lower cognitive score in the solid tumors group compared to hematological group.
\end{abstract}

Keywords: childhood cancer-PedsQL ${ }^{\mathrm{TM}} 3.0$ cognitive functioning scale

Cite This Article: Manal A. Shehata, Mohamed Fawzy, Mai S. Saleh, Zeinab M. Monir, and Hanafy Ahmed, "Exploration of Cognitive Functioning in a Pilot Sample of Childhood Cancer Patients in Egypt." Journal of Cancer Research and Treatment, vol. 3, no. 2 (2015): 19-24. doi: 10.12691/jcrt-3-2-1.

\section{Introduction}

Pediatric cancer cases that occur in developing countries contributes for more than $85 \%$ of the whole childhood cancer population and are expected to exceed $90 \%$ in the next two decades. [1] Worldwide, malignancies of the hematopoietic system are the largest subgroup of childhood cancers, accounting for $30 \%$ to $60 \%$ of all tumors. They are followed by tumors of the brain and nervous system ( $10 \%$ to $20 \%$ ), bone (3\% to $10 \%$ ) and liver (1\% to $3 \%)$ [2].

Generally speaking, unprecedented changes in diagnostic techniques, treatment methods and supportive care for childhood malignancies have occurred during the last decades. Consequently, management has improved and the mortality rates have decreased [1]. Higher survival rates in the different cancers have resulted in heightened focus on improving quality of life of survivors and reducing treatment-related late effects, including cognitive deficits [3].

Recent studies have reported difficulties in the abilities of cancer patients to remember, think, and concentrate [4].
This could be attributed both to the disease as well as treatment [5]. Moreover, little is still known about the effects of chemotherapy on cognitive functions [6]. Hence, cognitive competency as one of the crucial lifestyle factors merits more attention in childhood cancer patients, with serious investigation of the degree to which cognitive performance could be affected by the different treatment regiments as well as the expected role of the type and prognosis of the disease itself [7].

As for developing countries in particular, they suffer from geographic inequality in cancer treatment compared to those in countries with advanced health care systems [8,9]. Moreover, Incidence rates, pathology, and clinical characteristics of various cancers seem different in developing and developed countries mainly due to different environments, life styles, dietary habits, and hygienic conditions. [1] According to experts, it is high recommended to broaden research which involves cases in developing countries putting in mind that progress in pediatric cancer treatment in Western countries has been attributed to a great extent to the serious, successive and continuous cancer research [10].

Consequently, the present study aimed to assess cognitive functioning in a pilot sample of childhood 
patients with cancer in Egypt. And to investigate the predisposing factors to cognitive impairments including variables related to disease, treatment, and some sociodemographic characteristics.

\section{Patients and Methods}

\subsection{Patients}

A convenient sample of 67 pediatric cancer patients aged from 8 to 12 years- with established diagnosis at the pediatric Oncology Department of the National Cancer Institute of Egypt were included onto the present work. The study was explorative and recruited patients of either hematological or solid malignancies at different phases of their treatment. Parents of every included patient were asked to complete the PedsQL ${ }^{\mathrm{TM}} 3.0$ Cognitive Functioning Scale [11] (Arabic versions), as well as a separate sheet in which maternal education, residence (urban or rural), family size, age at diagnosis and gender were included. Control group consisted of 37 healthy subjects from the same age group and were subjected to the same methodology.

\subsubsection{Instrument}

The parent proxy-report for children aged 8-12 years of the PedsQL ${ }^{\mathrm{TM}}$ 3.0 Cognitive Functioning Scale is a 6 item scale to assess different facets of cognitive functioning. A 5 point likert scale ranging from 0 for "never a problem" till 4 for "almost always a problem" was used to rank each item of the scale. A corresponding score from 0 to 100 is then calculated, where 0 represents the worst state of cognitive functioning and 100 represents the best one. Finally, the average of the scores for all items represents the total score of cognitive functioning.

\subsubsection{Linguistic Translation}

Items of the PedsQL ${ }^{\mathrm{TM}}$ 3.0 Cognitive Functioning Scale were translated into Arabic (native language of the study sample) using the PedsQL ${ }^{\mathrm{TM}}$ Measurement Model Translation Methodology. Linguistic validation process included forward translation from the source language (English) to the target language (Arabic), backward translation to the source language, and cognitive debriefing according to PedsQL ${ }^{\mathrm{TM}}$ Cognitive Interviewing MethodologySM [12,13]. A report for every step throughout the whole process was sent to Mapi research Institute in Lyon, France, on behalf of Dr. James W. Varni, the copyright owner of the questionnaire for approval that was successfully issued.

\subsection{Measures}

\subsubsection{Intensity of Treatment}

Intensity of treatment received by patients of the study sample was rated on a 3-point scale, with $1=$ low: surgery only or six months chemotherapy only or both, with a favorable prognosis; 2 = medium: treatment longer than 6 months according to the treatment protocol, with an intermediate prognosis; 3 = high: treatment according to intensive protocols, bone marrow transplantation, with unfavorable prognosis $[14,15]$.
Phase of treatment; patient actively receiving therapy at any phase of the treatment protocol were defined as ontreatment group. Whereas, the follow-up group indicated patients who ended their treatment and were scheduled for timed interval follow up.

Therapy duration; short if $\leq 6$ month, between 6 month and 1 year was medium, and $\geq 1$ year defined as long duration of therapy.

Length of hospital admission; had 3 levels: low for admission periods within one third of the total duration of therapy, medium if admission period was more than one third and less than 2 thirds of the total duration, and high if it exceeded 2 thirds the total treatment duration.

\section{Statistical Analysis}

Score of the Cognitive Functioning Scale for each participant was calculated according to the guidelines of the PedsQL ${ }^{\mathrm{TM}}$ Cognitive Functioning Scale developer. Descriptive statistics were expressed as mean, median, and standard deviation. Data analysis was performed using the student t-test for the comparison between two groups, one way ANOVA was used for multiple group comparison, and a value of $\mathrm{p}<0.05$ was considered significant. Analyses were conducted using SPSS (SPSS Inc., Chicago, IL, with SPSS version 18).

\section{Results}

In order to obtain discriminative values with apparent significance the study sample of childhood cancer patients is divided into two groups according to the type of disease; group 1 for patients with hematologic diseases and group 2 for patients with solid tumors. Group 3 is represented by normal controls of age matched children. Demographic information and other descriptive data for the study groups are provided in Table 1. Hematologic cancers are represented by ALL (35/47), non Hodgkin lymphoma (5/47) Hodgkin lymphoma (4/47) and AML (1/47). For the solid tumors, $25 \%$ have brain tumors, $25 \%$ suffer from Ewings sarcoma and 15\% are diagnosed for MB. Study patients were 33 males and 14 females in the hematologic group with a median age of 7.5 years at diagnosis and 5.02 as mean value for family size. While in the solid tumor group there were 10 males and 10 females with a median age of 7.9 years at diagnosis and 5.8 as mean value for family size. There was no difference between the groups with regard to the age at diagnosis $(\mathrm{t}=-0.5, \mathrm{p}=0.6)$ and family size $(\mathrm{F}=2.1, \mathrm{p}=0.12)$. The urban/rural distribution in the three groups is as follows: $54 \% / 46 \%, 57 \% / 43 \%$ and $68 \% / 32 \%$ for the hematologic, solid tumors and control groups, respectively. Some maternal education is shown for $59 \%$ in the hematologic group, 50\% in the solid group and $90 \%$ in the control group. The on-treatment and follow-up phase of treatment show very close percentages in both hematologic (53\% and 47\%) and solid tumor groups (55\% and 45\%). High therapy duration of 1 year or above at time of evaluation prevails in both hematologic and solid tumor groups and represents $51 \%$ and $50 \%$ of study sample in the 2 groups, respectively. As for the Cognitive Functioning Scale mean score, it showed to be 
85.1, 69.6 and 83.2 for the hematologic, solid tumor and control groups, respectively.

Table 1. Socio demographic, cancer and treatment variables in patients and controls

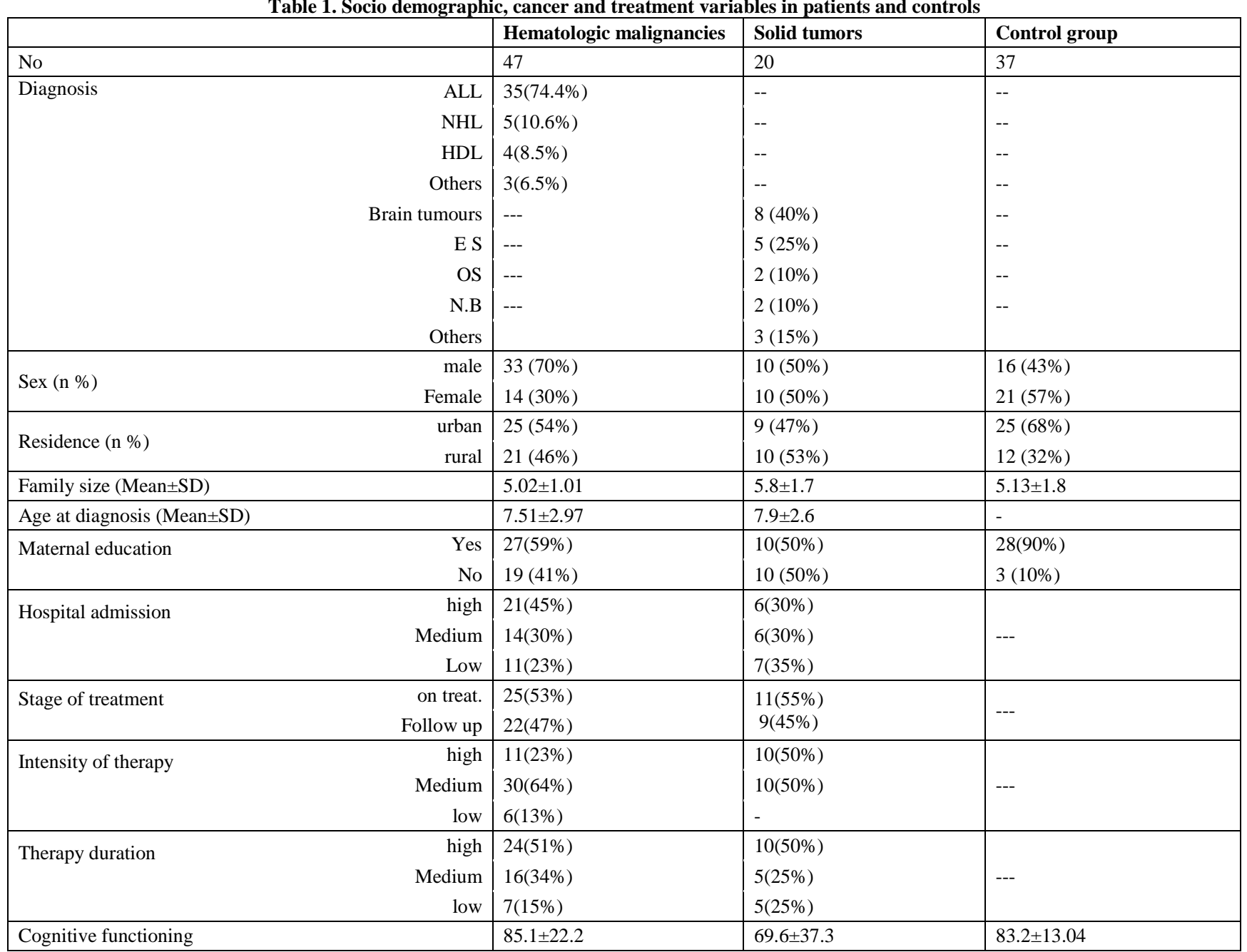

ALL= Acute Lymphoblastic Leukemia; NHL=Non Hodgkin Lymphoma; HD=Hodgkin Lymphoma; ES=Ewing's sarcoma; OS=Osteosarcoma; $\mathrm{NB}=$ Neuroblastoma.

As shown in (Figure 1) the comparison between the three studied groups (hematological malignancies, solid tumors and control group) as regard their cognitive functioning score revealed that solid tumor group had the lowest score ( $\mathrm{p}$ value $=0.04)$. Moreover, statistically significant difference was shown upon comparing the hematologic group and solid tumor group $(\mathrm{t}=2.1$, $\mathrm{p}$ $=0.038$ ), and again between solid tumor group and control subjects $(p=0.047)$ revealing in both cases declined cognitive functioning within patients with solid tumors.

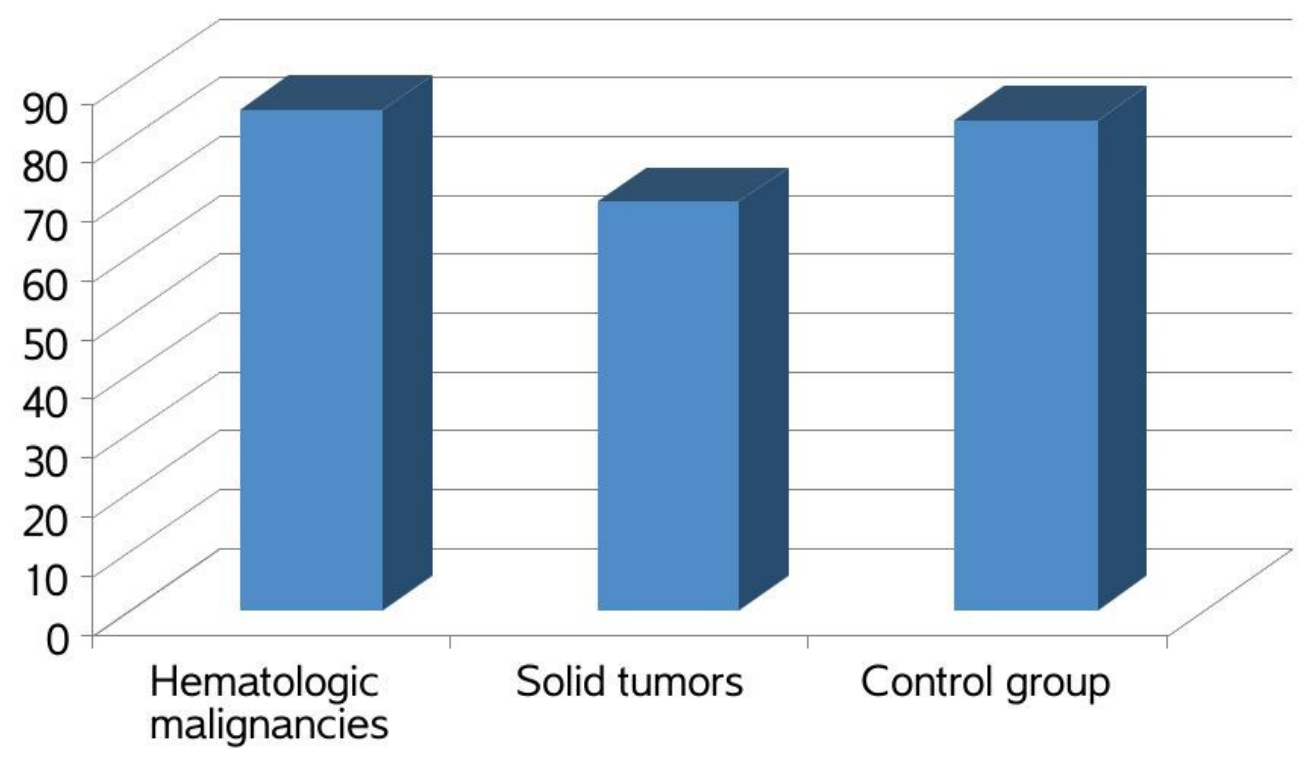

Figure 1. Mean cognitive functioning score of the studied groups, showing a lower score for the solid tumour group than the other two groups, p value $($ ANOVA $)=0.04$ 
Table 2 shows the impact of some socio-demographic variables on cognitive functioning score. To search the effect of the age at diagnosis on cognitive functioning, cases were divided into two subgroups: (i) the first group: children younger than or equal to 5 years and (ii) the second group: children older than 5 years at the time of diagnosis. As shown by the results the second group (older than five years) was associated with lower cognitive functioning score (69.3) in the solid group compared to the score (86.7) for group with hematological malignancies at the same age category. Urban residence also significantly affected the cognitive functioning score (51.84) of the solid group which showed to be lower than scores of the other two groups; the hematological malignancies and normal control (83.98 and 85.3, respectively) with a level of significance $=0.001$. Negative maternal education was another factor that negatively affected the scoring of the solid group (67.08) in comparison to hematologic group (89.9) with p value of 0.03 using student $t$ test (not mentioned in the table). To look for the effect of sex, cognitive functioning score for males and females were analyzed separately, but no statistically significant differences were found between the groups. Also, there was no significant difference between the groups in their cognitive functioning score in relation to their family size.

Table 2. Effect of some studied socio demographic variables on cognitive functioning score of the studied groups

\begin{tabular}{|c|c|c|c|c|c|}
\hline & & Hematologic & Solid & control & p-value \\
\hline & & \multicolumn{3}{|c|}{ Mean \pm SD(n) } & \\
\hline \multirow[t]{2}{*}{ Age at diagnosis } & $0-5$ & $81.23 \pm 22.57$ (14) & $69.43 \pm 29.25(3)$ & & 0.4 \\
\hline & $>5$ & $86.7 \pm 22.2(33)$ & $69.6 \pm 39.32(17)$ & ---- & $0.05^{*}$ \\
\hline \multirow[t]{2}{*}{ Sex } & male & $83.32 \pm 22.64$ (33) & $71.23 \pm 39.79(10)$ & $84.6 \pm 10.4(16)$ & 0.3 \\
\hline & Female & $89.27 \pm 21.49(14)$ & $67.92 \pm 36.74(10)$ & $82.1 \pm 14.9(21)$ & 0.09 \\
\hline \multirow[t]{2}{*}{ Residence } & urban & $83.98 \pm 21.51(25)$ & $51.84 \pm 39.25(9)$ & $85.3 \pm 14.5(25)$ & $0.001^{* *}$ \\
\hline & rural & $85.9 \pm 23.96(21)$ & $82.49 \pm 31.1(10)$ & $78.8 \pm 8.00(12)$ & 0.7 \\
\hline \multirow[t]{2}{*}{ Family size } & $0-4$ & $83.6 \pm 25.6(12)$ & $60.00 \pm 54.7(5)$ & $86.97 \pm 16.7(8)$ & 0.2 \\
\hline & $>4$ & $85.58 \pm 21.33(35)$ & $72.76 \pm 31.44(15)$ & $81.1 \pm 12.4(23)$ & 0.3 \\
\hline \multirow[t]{2}{*}{ Maternal education } & Yes & $81.77 \pm 24.52(27)$ & $72.07 \pm 40.06(19)$ & $81.7 \pm 13.8(28)$ & 0.8 \\
\hline & No & 89.9 $\pm 18.8(19)$ & $67.08 \pm 36.3(10)$ & $91.6 \pm 8.4(3)$ & 0.07 \\
\hline
\end{tabular}

* Significant t- test $\mathrm{p}<0.05 \quad * *$ Highly significant one-way (ANOVA) $\mathrm{p}<0.01$.

Table 3 shows the impact of some treatment variables solid tumors group than the scores (83.83 and 86.1 , on cognitive functions of cancer childhood patients as compared between hematologic and solid cancer groups. High therapy duration as well as long duration of hospital admission were associated with a lower cognitive functioning score (56.66 and 51.38, respectively) in the respectively) in the hematological group $(\mathrm{p}<0.05)$, while different intensities of therapy and the stage of treatment although negatively affected scores in patients with solid tumors compared to those with hematological malignancies, yet such differences were not significant.

Table 3. The impact of cancer treatment variables on cognitive functioning score

\begin{tabular}{|c|c|c|c|c|}
\hline & & Hematologic & Solid & p-value \\
\hline & & \multicolumn{2}{|c|}{ Mean \pm SD (n) } & \\
\hline \multirow[t]{3}{*}{ Intensity of therapy } & high & $82.56 \pm 23.63(11)$ & $64.16 \pm 38.3(10)$ & \\
\hline & Medium & $83.18 \pm 23.31(30)$ & $74.99 \pm 37.52(10)$ & 0.2 \\
\hline & low & $99.30 \pm 1.71(6)$ & ------ & 0.4 \\
\hline \multirow[t]{3}{*}{ Therapy duration } & high & $83.83 \pm 20.65(24)$ & $56.66 \pm 39.67(10)$ & $0.01^{* *}$ \\
\hline & Medium & $83.5 \pm 27.58(16)$ & $95.00 \pm 11.18(5)$ & 0.38 \\
\hline & low & $92.84 \pm 13.14(7)$ & $69.98 \pm 41.49(5)$ & 0.19 \\
\hline \multirow[t]{3}{*}{ Hospital admission } & high & $86.1 \pm 23.84(21)$ & $51.38 \pm 45.46(6)$ & $0.017^{*}$ \\
\hline & Medium & $83.32 \pm 23.58(14)$ & $83.32 \pm 12.08(6)$ & 1.00 \\
\hline & low & $87.11 \pm 19.24(11)$ & $69.04 \pm 43.49(7)$ & 0.24 \\
\hline \multirow[t]{2}{*}{ Stage of treatment } & on treat. & $87.82 \pm 22.41(25)$ & $75.74 \pm 31.7(11)$ & 0.19 \\
\hline & Follow up & $81.99 \pm 22.14(22)$ & $62.03 \pm 43.96(9)$ & 0.1 \\
\hline
\end{tabular}

* Significant t- test $\mathrm{p}<0.05 \quad$ **highly significant- test $\mathrm{p}<0.01$.

\section{Discussion}

Deteriorated cognitive functioning in children with cancer could be attributed either to cancer per se and/or cancer treatments -including chemotherapy- as described by Vardy et al [16]. The current study compared -parentreported- cognitive functioning between normal children and two different groups of childhood malignancies; solid tumors and hematological cancers. Patients with solid tumors (40\% brain tumors) showed significantly lower cognitive functioning score compared to those with hematologic malignancies and the healthy control which may suggest a prominent role for solid tumors -in particular- in cognitive dysfunction reported in childhood cancer patients. This shows to be consistent with research results recording early cognitive impairment in children with brain tumors sometimes even prior to radiotherapy/chemotherapy treatment [17]. Earlier studies which reported cognitive impairment in $15 \%-50 \%$ of adult solid tumor survivors could also suggest some kind of serious role of the type of disease (solid tumors) in affecting cognitive state of cancer survivors $[18,19,20]$.

On the contrary, group representing hematological cancers in the current study -in which ALL constituted the largest proportion (74.4\%) of its composition- showed matched results to normal control in the cognitive 
functioning scale which means that neither the disease nor its treatment affected cognition in the represented sample. This goes in agreement with some studies on ALL childhood patients [21].

As for chemotherapy in specific and its effect on cognitive function of childhood cancer patients, although several studies [18,19,20,22] have found evidence supporting the influence of chemotherapy on cognitive functioning, yet negative studies have also been reported. $[23,24,25,26,27]$ This discrepancy may be due to one or more of factors reported by Vardy et al. [16] which included the different chemotherapy regimens administered, variations in normative data and reference groups, statistical cut-offs used to define cognitive impairment and others. Moreover, Becham (1988, 1989) reported that cognitive dysfunction due to chemotherapy may not appear immediately after treatment but needs at least from 2 to 3 years after treatment cessation which is not the case in the study sample $[28,29]$.

In addition to the effect of the type of cancer and the role of chemotherapy, number of variables investigated in our study showed to contribute significantly to the deterioration of cognitive functioning in pediatric patients with solid tumors compared to patients with hematological malignancies and/or normal control. The indicated variables included treatment related variables like long therapy duration and excessive hospital admission; that agrees with Whittington (2007), who reported that, the time since treatment is an important indicator of risk for cognitive problems [30] together with length of hospital admission which is known to affect quality of life of cancer patients in general.

In consistence with literature [31], results of the current study also showed some host factors to influence the neurocognitive outcomes. Such factors included older age at diagnosis, urban residence and illiterate mothers which were associated with a lower cognitive functioning score in the solid tumors group compared to hematological group. Young age at diagnosis -generally- was demonstrated to be a risk factor for cognitive dysfunction in children cancers over time [30] with reported higher risk for cognitive sequelae in patients younger than 6 to 8 years [32] as it is crucial time for cognitive development. In the present study all of our patients belonged to the high risk group with respect to age at diagnosis, yet our results showed patients with solid tumors to have their cognitive function much more deteriorated than the other group with hematological diseases. Such a decrease was significant in the group with age older than 5 years at diagnosis and could be explained -in our considerationby the suggested frequent school absence. Non significant decrease in the group with age less than 5 years at diagnosis on the other hand may be due to the small number of patients and the subsequent large value of SD.

Negative maternal education in our study also showed to be significantly associated with worse cognitive functioning score in patients with solid malignancies in accordance with other studies [33]. Children with solid cancers living in rural regions did not show significant difference in the cognitive functioning scale score compared to patients with hematological diseases. On the other hand urban residence showed to affect cognitive functioning negatively in patients with solid tumors. According to Wells [31] window views onto green vegetation, rather than bare dirt or pavement were associated with increased cognitive function in lowincome children generally and this could explain why children with solid tumors in urban residence showed significantly more deteriorated cognitive functioning scores than those in rural areas. In contrast to several studies which revealed that girls perform more poorly than boys in cognitive tests ${ }^{[21,35]}$ negligible effect of sex was found in the present work.

\section{Conclusion}

In conclusion, there is a crucial need of cognitive function testing at the time of cancer diagnosis as a baseline as well as the different stages of disease and treatment to clarify the specific role of the tumor type, surgery, type of treatment and medical procedures in the possible cognitive decline of children with different types of cancers. After the current work, we can suggest that the parent-proxy Ped'sQL Cognitive functioning Scale could be a potential screening measure for cognitive function in childhood cancer patients as it was able to discriminate between diseased and healthy children as well as between patients with solid and hematological diseases with respect to cognitive functioning. More detailed neuropsychological tests are needed for specific information about the nature and severity of cognitive dysfunction at different loci throughout the length of the treatment period and even for long term survivors as a second step to provide a better view for specific issues related to cognitive dysfunction in childhood cancer patients. Besides, studies on big samples are also recommended to clarify the role of predisposing variables. Furthermore, development of interventions that address cognitive late effects is imperative, intervention studies can be more efficiently designed to target the underlying mechanisms to try and prevent cognitive impairment from occurring and to treat it once it has occurred.[16] Last but not least, developing countries represent a highly fertile medium for health research, there are very much low available data describing the health conditions of childhood cancer patients even on the level of preliminary screening studies.

\section{Conflict of Interest Statement}

The authors declare that they have no conflict of interest.

\section{References}

[1] Yaris N, Mandiracioglu A, Büyükpamukcu M. Childhood cancer in developing countries. PediatrHematolOncol 2004; 21:237-253.

[2] Steliarova-Foucher E, Hery C, Pisani P. Childhood cancer: rising to the challenge. UICC; Geneva. The burden of childhood cancer 2006; pp. 9-14.

[3] Conklin HM, Krull KR, Reddick W E, et al. Cognitive Outcomes Following Contemporary Treatment Without Cranial Irradiation for Childhood Acute Lymphoblastic Leukemia. J Natl Cancer Inst.2012; 104:1386-1395.

[4] Bender CM, Paraska KK, Sereika SM, et al. Cognitive function and reproductive hormones in adjuvant therapy for breast cancer: a critical review. J Pain Symptom Manage 2001; 21: 407-424. 
[5] Moore BD 3rd. Neurocognitive outcomes in survivors of childhood cancer. J PediatrPsychol 2005; 30(1):51-63.

[6] Jansen CE, Miaskowski C, Dodd M, et al. A metaanalysis of studies of the effects of cancer chemotherapy on various domains of cognitive function. CANCER 2005; 104 / 10: 2222-2233.

[7] Fardell JE., Vardy J, Johnston I N, et al. Chemotherapy and Cognitive Impairment: Treatment Options. Clinical pharmacology \& Therapeutics 2011; 90 NUMBER 3.

[8] Pui CH, Schrappe M, Masera G, et al. Ponte di Legno Working Group: statement on the right of children with leukemia to have full access to essential treatment and report on the Sixth International Childhood Acute Lymphoblastic Leukemia Workshop. Leukemia 2004; 18: 1043-1053.

[9] Pui CH, Ribeiro RC. International collaboration on childhood leukemia. Int J Hematol 2003; 78: 383-389.

[10] Ribeiro RC and Pui CH. Saving the Children - Improving Childhood Cancer Treatment in Developing Countries. N Engl J Med 2005; 352:2158-2160.

[11] Varni, JW, Limbers CA., Sorensen LG, et al. PedsQL ${ }^{\mathrm{TM}}$ Cognitive Functioning Scale in pediatric liver transplant recipients: Feasibility, reliability and validity. Quality of Life Research 2011; 20: 913-921.

[12] Varni JW, Burwinkle TM, Seid M, et al. The PedsQLÔ 4.0 as a pediatric population health measure: Feasibility, reliability, and validity. Ambulatory Pediatrics 2003; 3: 329-341.

[13] Sprangers M, Cull A, Groenvold $M$, et al. The European Organization for Research and Treatment of Cancer approach to developing questionnaire modules: An update and overview. Quality of Life Research 1998; 7: 291-300.

[14] Magal-Vardi O, Laor N, Toren A, et al. Psychiatric morbidity and quality of life in children with malignancies and their parents. The Journal of Nervous and Mental Disease 2004;. 92:872-875.

[15] Landolt MA, Vollrath M, Niggli FK, et al. Health-related quality of life in children with newly diagnosed cancer: a one year followup study. Health and Quality of Life Outcomes 2006; 4:63.

[16] Vardy J, Wefel JS, Ahles T, et al. Cancer and cancer-therapy related cognitive dysfunction: an international perspective from the Venice cognitive workshop. Annals of Oncology 2008; 19: 623-629.

[17] 14 Butler RW, Haser JK. Neurocognitive effects of treatment for childhood cancer mental retardation and developmental disabilities research reviews 2006;.12: 184-191.

[18] Brezden CB, Phillips KA, Abdolell M, et al. Cognitive function in breast cancer patients receiving adjuvant chemotherapy. J ClinOncol 2000;18: 2695-2701.

[19] Ahles TA, Saykin AJ, Furstenberg CT et al. Neuropsychologic impact of standard dose systemic chemotherapy in long-term survivors of breast cancer and lymphoma. J ClinOncol. 2002; 20: 485-493.

[20] Tchen N, Juffs HG, Downie FP et al. Cognitive function, fatigue, and menopausal symptoms in women receiving adjuvant chemotherapy for breast cancer. J ClinOncol 2003; 21: 4175-4183.
[21] Von derWeid N, Mosimann I, Hirt A, et al. Intellectual outcome in children and adolescents with acute lymphoblastic leukaemia treated with chemotherapy alone: Age- and sex-related differences. Eur J Cancer 2003; 39: 359-365.

[22] Castellon SA, Ganz PA, Bower JE, et al. Neurocognitive performance in breast cancer survivors exposed to adjuvant chemotherapy and tamoxifen. J. Clin. Exp. Neuropsychol. 2004; 26: 955-969.

[23] Jenkins V, Shilling V, Deutsch G, et al. A 3-year prospective study of the effects of adjuvant treatments on cognition in women with early stage breast cancer. Br J Cancer. 2006; 94(6): 828-34.

[24] Donovan KA, Small BJ, Andrykowski MA, et al. Cognitive functioning after adjuvant chemotherapy and/or radiotherapy for early-stage breast carcinoma. Cancer. 2005; 104(11): 2499-507.

[25] Copeland DR, Fletcher JM, Pfefferbaum-Levine B, et al. Neuropsychological sequelae of childhood cancer in long-term survivors. Pediatrics.1985; 75:745-753.

[26] Butler RW, Hill JM, Steinherz PG, et al. Neuropsychologic effects of cranial irradiation, intrathecal methotrexate, and systemic methotrexate in childhood cancer. J Clin Oncol.1994; 12: 2621-2629.

[27] AndersonV, Godber T, Smibert E, et al. Neurobehaviouralsequelae following cranial irradiation and chemotherapy in children: An analysis of risk factors. PediatrRehabil. 1997; 1:63-76.

[28] Peckham VC. Learning disorders associated with the treatment of cancer in childhood. Journal of the Association of Pediatric Oncology Nursing 1988; 5: 10-13.

[29] Peckham VC. Learning disabilities in long-term survivors of childhood cancer: Concerns for parents and teachers. Reading, Writing and Learning Disabilities 1989; 5: 313-325.

[30] Whittington E: Survivors at Risk for Cognitive Dysfunction. Cure, 2007

http://www.curetoday.com/index.cfm/fuseaction/article.PrintArticl e/article_id/111. Last accessed November, 2013.

[31] Nathan PC, Patel SK, Dilley K, et al.Guidelines for Identification of, Advocacy for and Intervention in Neurocognitive Problems in Survivors of Childhood Cancer. Arch PediatrAdolesc Med. 2007; 161(8): 798-806.

[32] Spencer J. The Role of Cognitive Remediation in Childhood Cancer Survivors Experiencing Neurocognitive Late Effects. Journal of Pediatric Oncology Nursing 2006; 23(6): 321-325.

[33] Vanderploeg RD, Schinka JA, Baum KM, et al. WISC-III premorbid prediction strategies: demographic and best performance approaches. Pychol Assess.1998; 10 (3):277-284.

[34] Wells NM. At home with nature: effects of "greenness" on children's cognitive functioning. Environ. Behav.2000; 32 (6):775-795.

[35] Buizer AI, de Sonneville LM, van den Heuvel-Eibrink MM, et al. Chemotherapy and attentional dysfunction in survivors of childhood acute lymphoblastic leukemia: Effect of treatment intensity.Pediatr Blood Cancer 2005; 45:281-290. 